\title{
HOT SPOT DETECTION AND SPATIO-TEMPORAL DYNAMICS OF DENGUE IN QUEENSLAND, AUSTRALIA
}

\author{
S. Naish ${ }^{\mathrm{a}, *}$, S.Tong ${ }^{\mathrm{a}}$ \\ ${ }^{a}$ School of Public Health and Social Work, Institute of Health and Biomedical Innovation, Queensland University of Technology, \\ Brisbane, Queensland, Australia - (s.naish@qut.edu.au)
}

\section{Commission VI, WG VI/4}

KEY WORDS: Clusters, Dengue, Geographical information systems, Spatiotemporal, Spatial autocorrelation

\begin{abstract}
:
Dengue has been a major public health concern in Australia since it re-emerged in Queensland in 1992-1993. This study explored spatio-temporal distribution and clustering of locally-acquired dengue cases in Queensland State, Australia and identified target areas for effective interventions. A computerised locally-acquired dengue case dataset was collected from Queensland Health for Queensland from 1993 to 2012. Descriptive spatial and temporal analyses were conducted using geographic information system tools and geostatistical techniques. Dengue hot spots were detected using SatScan method. Descriptive spatial analysis showed that a total of 2,398 locally-acquired dengue cases were recorded in central and northern regions of tropical Queensland. A seasonal pattern was observed with most of the cases occurring in autumn. Spatial and temporal variation of dengue cases was observed in the geographic areas affected by dengue over time. Tropical areas are potential high-risk areas for mosquito-borne diseases such as dengue. This study demonstrated that the locally-acquired dengue cases have exhibited a spatial and temporal variation over the past twenty years in tropical Queensland, Australia. There is a clear evidence for the existence of statistically significant clusters of dengue and these clusters varied over time. These findings enabled us to detect and target dengue clusters suggesting that the use of geospatial information can assist the health authority in planning dengue control activities and it would allow for better design and implementation of dengue management programs.
\end{abstract}

\section{INTRODUCTION}

Dengue is a growing international public health concern. Recent estimates indicate that 390 million people are infected with dengue across the globe with 96 million people suffering from dengue and more than 40,000 deaths each year (Bhatt et al., 2013). Dengue viruses (Flaviviridae) are transmitted to humans by mosquitoes of Aedes species. Aedes aegypti is the primary vector worldwide, and is predominantly found in tropical urban environments. It has a so-called cosmo-tropical distribution annually, and spreads to more temperate regions during the summer (Jansen et al., 2010). Aedes albopictus, is currently considered of secondary importance in dengue transmission, except in Asian countries, being present in rural or semi-urban habitats (Caminade et al., 2012, Honorio et al., 2009, Vezzani et al., 2008). Both species living near man are adapted to human habitation and survive on fresh water (Higa, 2011).

Aedes aegypti is the main vector of dengue virus in Queensland, Australia (Van Den Hurk et al., 2010). It is most active at daytime with peak activity around sunrise and sunset, and is often hidden inside homes or other dwellings. The female bites mainly humans and lays her eggs fundamentally in man-made containers (Russell, 2009b, Russell, 2009a). The average lifespan of the female mosquito is about 14-30 days and they can fly about $30-50 \mathrm{~m}$ per day on an average, indicating that they generally would move around $240-500 \mathrm{~m}$ range in their lifetime (Takahashi et al., 2005). This short flight range contributes to the limited local spread of the disease. Thus, this is the scale at which hotspots or clusters of dengue need to be identified in order to determine the overall transmission pattern. Dengue outbreaks have been identified to cluster at this scale in high resolution spatio-temporal analyses (Vanwambeke et al., 2011, Higa, 2011, Wen et al., 2010). Ae. albopictus is currently threatening the Australian mainland and has been detected on a number of Torres Strait islands (Williams et al., 2010 , Hanna, 2009). Unlike Ae.aegypti, Ae.albopictus is natural and artificialcontainer breeder. It is also a forest-fringe mosquito breeder (Russell, 1998). Unlike Ae.aegypti, it is highly adaptive, invasive and flexible in its behaviour. It chooses a habitat based on the availability of food resources and availability of locations for reproduction and development. Dengue outbreaks are only expected to become worse with the expansion of the habitats of these two mosquito species. The prime factors for this expansion are a combination of global human movement, changing climate and rapid urbanisation providing these mosquitoes with a greater chance of establishing in many countries across the globe (World Health Organisation, 2014, Cdc, 2014).

Historically, the first dengue outbreak has occurred in 1879 in Queensland in Australia (Hanna, 2009). Since 1885, 13 major dengue outbreaks have been documented in Queensland in the following areas: Thursday Island, Townsville, Cairns, Cooktown, Port Douglas, Charters Towers, Normanton, Mackay, Ingham and Bowen with cases inland at Hughenden,

\footnotetext{
* Corresponding author. This is useful to know for communication with the appropriate person in cases with more than one author.
} 
Barcaldine, etc. The first death caused by dengue occurred in Charters Towers in Queensland in 1885, and was followed by a large outbreak in 1887 with 60 recorded deaths, of which 30 were children (Queensland Health., 2014). Dengue outbreaks in Queensland have been increased in frequency and intensity since the early 1990s (Queensland Health., 2014). In the last decade, for example, in 2003 and 2004, there were six outbreaks in north Queensland with a combined total of nearly 900 cases from Cairns, Townsville and the Torres Strait. In Australia, outbreaks are a mix of locally-acquired and overseastransmitted cases. According to the Queensland Health, a dengue outbreak is declared when there is one or more locallyacquired dengue cases are confirmed. An overseas-transmitted limported case is defined as someone who is infected with dengue overseas (i.e., viraemic traveller) and arrives in Australia with the virus in their blood (Queensland Health., 2014). A locally-acquired case is defined as when a local dengue mosquito bites this overseas dengue infected person and it passes the virus on to other local people by biting them (Queensland Health., 2014). The number of dengue cases has declined substantially from 2004 to 2007 since the beginning of the Queensland wide Dengue Alert and Response Team and Dengue Management programmes in the early 2000 (Queensland Health, 2013). However, dengue still remains as a significant public health problem in northern Queensland, with approximately $50 \%$ dengue cases on average recorded in Australia annually (National Notificable Disease Surveillance System, 2014). The number of overseas-transmitted dengue cases in Queensland has increased significantly since 2007. In 2008 and 2009, there were five outbreaks in north Queensland with over 1000 recorded cases. In the recent years, evidence suggests that dengue has re-emerged within Queensland. For example, dengue has been recently re-identified in Townsville, Innisfail, Pimlico and Ingham (Queensland Health., 2014).

Spatial and temporal analysis of disease outbreaks is important to evaluate whether the occurrence of a disease is randomly distributed or tends to occur as clusters over time and/or space after adjusting for known confounding factors. This may provide clues for the etiology of the disease or inferences about the epidemiological determinants and eventually aid health surveillance services in policy-making decisions. Various spatio-analytical techniques are applied to identify spatial and temporal characteristics of disease including cluster detection, hotspot analysis, and regression models. Anselin compared the techniques using free software programs such as CrimeStat, GeoDa, SaTScan, and spatial analysis packages for use in the open source R program (Anselin, 2004). He confirmed that Kulldorff's spatial scan statistic (Kulldorff, 1997) and the Local Moran's I (Anselin, 1995) be used in conjunction for disease cluster analyses.

Due to the absence of specific treatment for dengue and vaccine, interventions are currently focussed on controlling the spread of vector populations by adapting genetically modified vector control methods, for example, the introduction of Wolbachia in northern Queensland and improved ovi-trap mechanisms (Estallo et al., 2011). To develop the effective vector control programmes, it is important to characterise the spatial and temporal characteristics of dengue. Hence, in this study, we identified spatial and space-time clusters of dengue in order to help future dengue surveillance, mosquito control policy analysis and develop effective dengue control programmes in north Queensland, Australia.

\section{METHODS}

\subsection{Study Area}

Queensland is the third largest state by population in Australia (after New South Wales and Victoria). It occupies an area of $1,723,936 \mathrm{~km}^{2}$ with a total population of 4.56 million people (approximately $20 \%$ of Australia's population) and is the fastest growing state with $23.9 \%$ of population growth in Australia (Australian Bureau of Statistics, 2013). Northern Queensland, a tropical region which is $100 \mathrm{~km}$ north of the Tropic of Capricorn is selected as the study area as it had the largest total number of recorded notifications $(n=2,327)$ in Queensland, and the largest compared to any other Australian state and territory, during the period 2005-2012 (National Notificable Disease Surveillance System, 2014). It has a tropical climate, with average temperatures ranging from 24-33 degrees Celsius in summer, and 14-26 in winter.

\subsection{Data Collection}

\subsubsection{Ethical statement}

The study was approved by both the Queensland Health $(\mathrm{QH})$ and Queensland University of Technology Human Research Ethics Committees. The population data were obtained from Australian Bureau of Statistics.

\subsubsection{Dengue data}

Dengue is a notifiable disease in Australia and all laboratorydiagnosed positive cases are required to be reported to the state government of QH, by the law of Public Health Act 2005. These records are archived by the Data Custodians, Communicable Disease Branch (CDB) unit in $\mathrm{QH}$ under the National Notifiable Disease Surveillance System.

A computerised locally-acquired dengue case notification dataset from January 1993 to June 2012 (approximately 20 years) for the study area was acquired from $\mathrm{CDB}, \mathrm{QH}$. A total of 2,327 locally-acquired dengue cases were obtained in Microsoft Excel format including date of notification, age group (e.g., 0-4; 5-9;10-14 etc.), gender, post code of residence and name of statistical local area (SLA) (i.e., Australian census unit).

\subsubsection{Population data}

Population data for each of the SLAs for the national census years 2001, 2006 and 2011 were obtained from the Australian Bureau of Statistics. We have adjusted SLA boundaries to match earlier censuses due to the latest SLA boundaries. For the other years during 1993 to 2012, the annual population data were estimated based on linear interpolation.

\subsection{Statistical Analysis}

In all the analyses, the study period was divided into four time periods, with five years in each time period for the ease of data analysis and to visualise the temporal patterns: Period 1: 19931997; Period 2: 1998-2002; Period 3: 2003-2007; and Period 4: 2008-2012. Population data for each period were attached to each SLA in the maps and these were used as the denominator in the computation of incidence rates. 
To fully investigate the presence of dengue clustering in north Queensland, we used two cluster detection programs: GeoDa (version 0.9.5-i, Urbana-Champaign, IL) which generates Anselin's Local Moran I (Local Indicators of Spatial Association, LISA) test statistics and SaTScan (version 6, Boston, MA) which implements Kulldorff's spatial scan statistic. Both these approaches test for spatial clustering using area (case and population at-risk) data, and output the location, approximate size and significance level of identified clusters. Different spatial analytical methods may identify different underlying spatial patterns (Moore et al., 1999). Consistent findings using more than one method, therefore, would suggest robust results. Period-wise distribution maps were produced using dengue cases and incidence rates by SLA. MapInfo Professional was used to produce the final outputs such as tables and maps.

\subsubsection{Anselin's Local Moran I test}

We applied the GeoDa cluster detection software program for generating Anselin's Local Moran I (LISA) test statistics. The Local Moran I statistic assesses spatial autocorrelation and identifies administrative units (i.e., SLA s) with dengue rates statistically similar to and dissimilar from their neighbours. The null hypothesis is that there is no association in dengue incidence rates among neighbouring SLAs. The alternative hypothesis is that spatial clustering exists (neighbouring SLAs have similar dengue rates). We defined neighbours using queen contiguity relationships (single point) and obtained Monte Carlo p-values based on 9,999 conditional randomizations. Because of multiple comparisons and chances for overlapping clusters, we used an alpha level of 0.05 to assess statistical significance.

The spatial autocorrelation of expected incidence rates of dengue in four different time periods was assessed using Moran's I statistic. Spatial autocorrelation was considered significant if the $\mathrm{p}<0.05$. Moran's I ranges from 0 to 1 ; a value close to 0 indicates spatial randomness, and a positive value indicates positive spatial autocorrelation and a negative value denotes a negative spatial autocorrelation.

Local Indicators of Spatial Association (LISA) was used to describe the spatial patterns of dengue hot spots /clusters at the SLA level during the different study periods. LISA was used to identify significant hot spots (High-High), cold spots (LowLow), and spatial outliers (High-Low and Low-High) by calculating local Moran's I between a given location and the average of neighbouring values in the surrounding locations (Anselin, 1995). To identify the spatial patterns of dengue, LISA analysis was performed independently for the annual average incidence of dengue at the SLA level in each period using MapInfo Professional.

\subsubsection{Spatial scan statistics - SaTScan}

We applied spatial and space-time scan statistical analyses (Kulldorff, 1997) using SaTScan (version 8.02) program (Kulldorff, 2009), to test for the presence of statistically significant spatial and temporal clusters of dengue. We have conducted analysis including the total period and period-wise. The scale at which the data will be aggregated is an important consideration for the identification of clusters. Usually, the exact coordinates, zip-codes, blocks, census units, districts, cities or states are used as aggregation units in scan statistics (Kulldorff, 1997). Our analysis was performed using area-based data aggregated at the SLA level in Queensland. Because of its ability of both identifying localised clusters and evaluating their significance, the spatial scan statistics becomes more prominent relative to other statistical methods for disease clustering. Space-time scan statistics have been applied previously in several mosquito-borne diseases such as malaria (Coleman et al., 2009), Japanese encephalitis (Impoinvil et al., 2011) and Barmah Forest virus (Naish et al., 2011). However, this study is the first attempt to apply SaTScan on dengue in Australia.

The spatial scan statistic uses a scanning window that moves across space. For each location and size of the window, the number of observed and expected cases is counted, and the window with the greatest ratio of observed to expected cases is recorded. The total number of expected cases is calculated by considering an even distribution of cases across the population.

Purely spatial analysis: In SaTScan, cases files, population files, and coordinate files were used as inputs in this analysis. A Poisson-based spatial scan statistic models were used, where the number of events in an area was Poisson distributed according to a known underlying population at risk (Kulldorff, 1997). A circular window was created by letting the centre of the circle move over the study area. The window included different sets of neighbouring areas at different positions in the study area. For example, if the window contained the centroid of the area, then that whole area was included in the window. The centre of the window was positioned at the centroids of each SLA (the latitude/longitude information of geometric centre was obtained using MapInfo geocoding function). The circular window radius varied continuously from zero up to a maximum for each centroid of the circle. Thus, the window did not include more than a specified percentage of the total population at risk. Constraints on the maximum radius of the window could also be set. In this approach, the circular window was flexible both in location and size. Each circle was noted as a potential cluster. For each potential cluster, a likelihood ratio test statistic (Kulldorff, 2009) was used to determine if the number of observed dengue cases within the potential cluster was significantly higher than expected or not. The circle with the maximum likelihood ratio test statistic was defined as the most likely/primary cluster, indicating that it was least likely to have occurred by chance. The maximum observed value of the test statistic for each potential cluster was then compared with the overall distribution of maximum values. SaTScan identified the most likely (primary) clusters and assigned p-values on the basis of 9999 Monte Carlo replications (Kulldorff, 2009). The presence of significant clustering was also determined. In addition to the most likely cluster, secondary clusters were also identified and ranked based on their likelihood ratio test statistic. For this analysis, outbreaks were indicated by the number of dengue cases and the maximum spatial cluster size was set to include up to $50 \%$ of the population at risk in the scanning window.

Space-time analysis: For determination of factors affecting the distribution of outbreaks, space-time cluster detection is a more accurate technique compared to purely spatial scan, as it assesses the two dimensions simultaneously, avoiding multiple tests bias (Kulldorff, 2009). A cylindrical window with a circle indicating a geographic base and with height representing to time was used in the space-time scan statistic. Similar to purely spatial scan statistic, the base of the cylinder indicated the space, and the height represented the time period of the potential clusters. The cylindrical window was then moved in space and time, and therefore it examined each potential geographical location and size as well as each likely time 
period. A large number of overlapping cylinders of different size and shape, together covering the entire study area were constructed, where each cylinder represented as a potential cluster. The likelihood ratio test statistic was calculated in the same way as for the purely spatial scan statistic for calculating the likelihood for each window. Initial analysis was conducted including maximum spatial cluster size of $50 \%$ of the total number of cases in the temporal window.

Sensitivity analysis assessment: Comparative sensitivity analyses were performed for the purely spatial analysis using different maximum spatial cluster sizes. Comparative sensitivity analyses were also performed for the space-time analysis using a range of cluster sizes and circle radii. This is because the default setting of $50 \%$ used by SaTScan seemed unrealistic as indicated by other authors (Chen et al., 2008). Furthermore, climate and landscape characteristics are believed to strongly influence dengue transmission, and if a large population threshold (> $30 \%$ ) was used numerous functional ecological zones controlled by climate and landscape factors would be crossed by the area within that threshold. Attempts were made to find the most meaningful population thresholds from the range tested by analysing quantitatively and visualizing the resulting clusters.

\section{RESULTS AND DISCUSSION}

\subsection{Epidemic curves and outbreaks}

Figure 1 shows the epidemic pattern of dengue cases and incidence rates (Figure: 1) during 1993-2012. Dengue transmission fluctuated during the study period with two major epidemic peaks in 2003 and 2009 (653 cases and 945 cases, respectively). Peaks in incidence cases coincided with high monthly numbers of SLA areas with dengue cases.

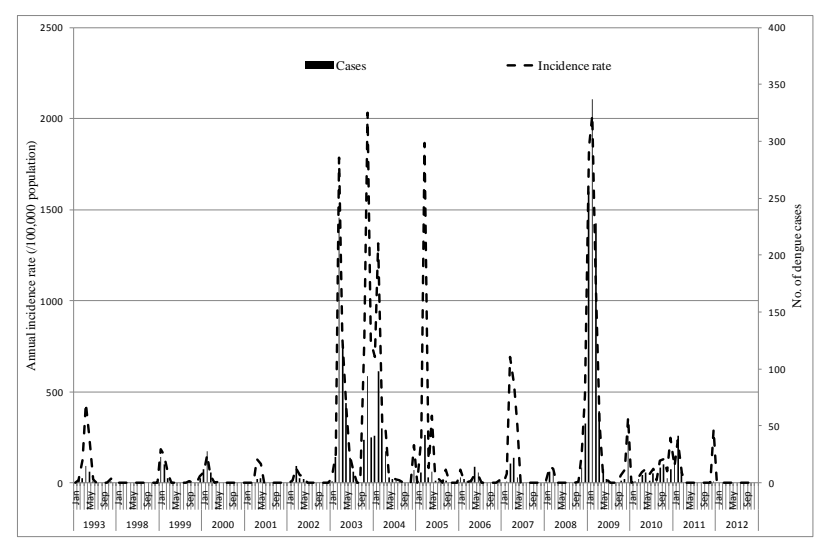

Figure 1: The epidemic pattern of dengue monthly cases and annual incidence rates

Table 1 indicates the descriptive statistics for dengue incidence rates among SLAs in northern Queensland for four time periods Period 1, period 2, period 3 and period 4 . There is a clear trend of geographic expansion of dengue transmission in northern Queensland through periods 1 and 2 (with mean incidence rates equal to 10.2 and 27.1 , respectively).

\begin{tabular}{|c|c|c|c|c|c|c|c|}
\hline Period & Mean & SD & Min & Q 1 & Med & Q 3 & Max \\
\hline 1 & 34.9 & 15.3 & 1.5 & 23.4 & 39.3 & 48.1 & 61.5 \\
2 & 10.2 & 11.6 & 3.2 & 5.4 & 5.46 & 9.4 & 61.5 \\
\hline
\end{tabular}

\begin{tabular}{|l|l|l|l|l|l|l|l|}
3 & 27.1 & 55.4 & 1.5 & 5.4 & 13.6 & 27.6 & 549.4 \\
4 & 12.3 & 30.7 & 1.5 & 4.6 & 5.4 & 13.6 & 671.1 \\
\hline
\end{tabular}

Table 1: Descriptive statistics of dengue incidence rates among statistical local areas in northern Queensland, 1993-2012

\subsection{Spatial autocorrelation}

Table 2 reveals a significant positive spatial autocorrelation of dengue incidence for all four time periods, where Moran's $I$ value was $0.036(\mathrm{p}<0.003)$ during period $1,0.061(\mathrm{p}<0.003)$ during period 2, $0.105(\mathrm{p}<0.001)$ during period 3 and 0.250 $(p<0.003)$ during period 4 . This indicates that nearby SLAs tend to have more similar baseline incidence rates than those further apart.

\begin{tabular}{|l|l|l|l|}
\hline $\begin{array}{l}\text { Study } \\
\text { period }\end{array}$ & Moran's & $\begin{array}{l}\text { Expected Moran's } \\
\text { I }\end{array}$ & I -value \\
\hline 1 & 0.0367 & -0.0021 & 0.003 \\
2 & 0.0617 & -0.0022 & 0.003 \\
3 & 0.1054 & -0.0021 & 0.001 \\
4 & 0.2508 & -0.0021 & 0.003 \\
\hline
\end{tabular}

Table 2: Spatial autocorrelation analysis

\subsection{Spatial clustering}

3.3.1 Anselin's Local Moran I test using GeoDa: Two statistically significant multi-centred (SLAs) clusters of dengue were identified (Figure 2). A single large cluster was identified in Cairns central region with five 5 locations in Cairns: $\mathrm{Mt}$ Whitfield, Cairns City, Cairns-Barron, Northward-Castle Hill and Railway Estate. The second high-risk cluster was detected in McKinlay shire region. The area of low- risk was located at Richmond region.

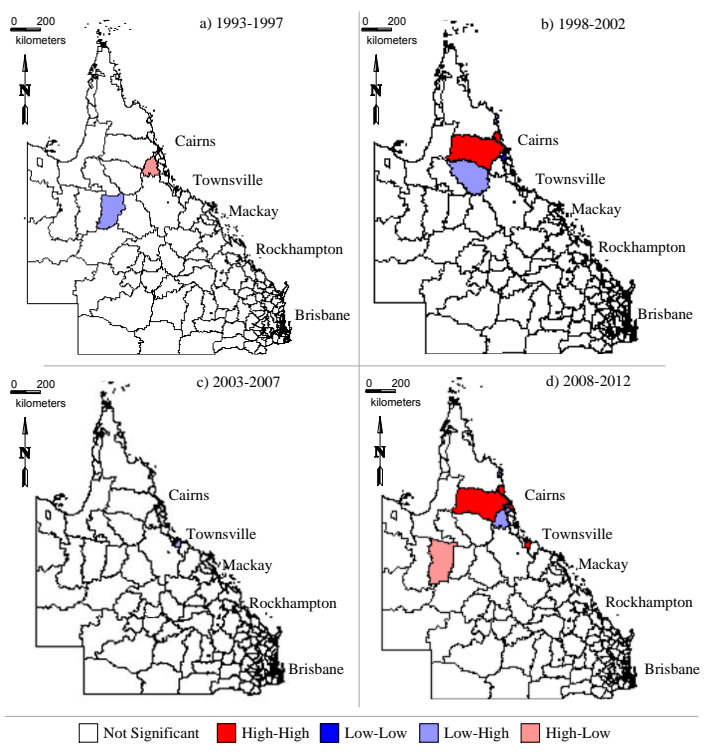

Figure 2: Spatio-temporal clustering of dengue using LISA test for the study period: a) 1993-1997, b) 1998-2002, c) 2003-2007 and d) 2008-2012 
3.3.2 Spatial scan statistics using SatScan: Using the maximum spatial cluster size of $10 \%$ of the population at risk, SaTScan identified a most likely statistically significant cluster encompassed three SLAs (Figure 3). The relative risk (RR) within the cluster was 168.8 with an observed number of 19 cases notified during period 1 , compared with 0.2 expected cases $(\mathrm{R}=298 \mathrm{~km})$. Another statistically significant secondary cluster with six SLAs was also identified in the same period $(\mathrm{RR}=17.4)$. During period1, interestingly, the cluster size $(\mathrm{R}=190 \mathrm{~km})$ was decreased with an increased number of SLAs $(n=4)$ from different areas. Similarly, during period 4 and 4 , the cluster size $(844 \mathrm{~km}$ and $596 \mathrm{~km}$ radius, respectively) was increased with inclusion of more SLAs (18 and 40 SLAs, respectively) from far northern and central regions.

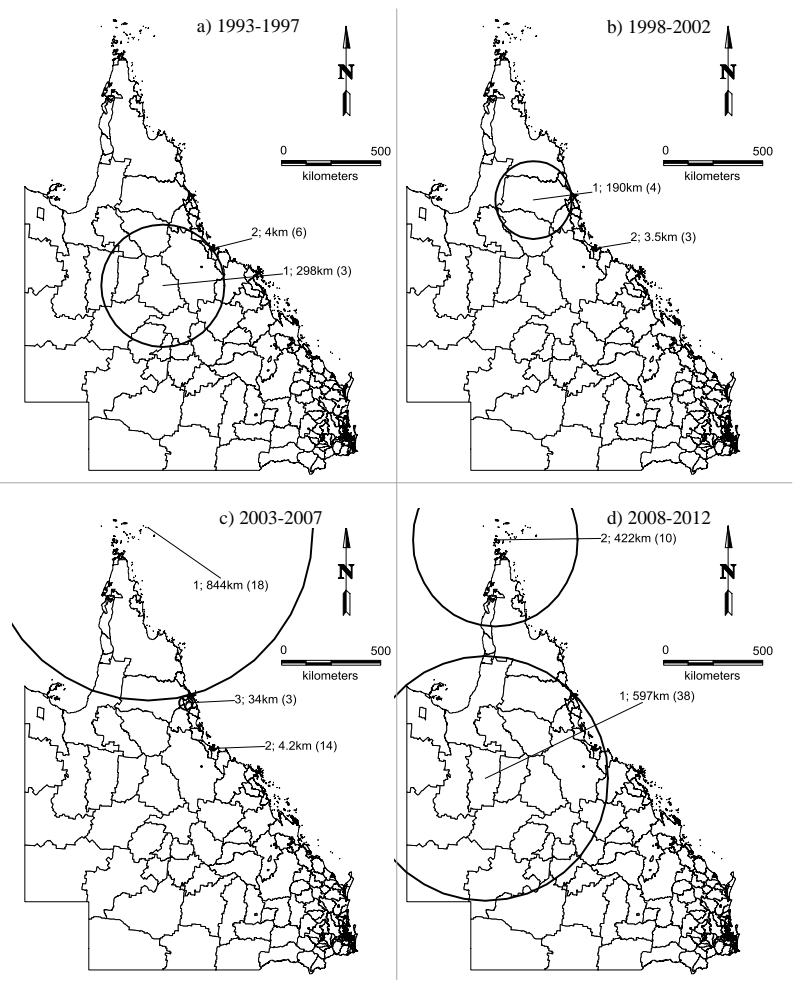

Figure 3: Spatio-temporal clustering of dengue using SatScan test for the period: a) 1993-1997, b) 1998-2002, c) 2003-2007 and d) 2008-2012

Table 3 shows the number of SLAs included in each cluster, radius $(\mathrm{km})$, observed cases, expected cases, RR and loglikelihood ratio for each time period. Very similar results were obtained when analysis was conducted using a spatial cluster size of $50 \%$ of population in each SLA (see Figure 3).

The results of the space-time analysis are generally similar to the purely spatial analysis in that the SLAs in the most likely cluster from the purely spatial analysis were also statistically significant in the space-time analysis during four time periods. Using a spatial cluster size of $10 \%$ of the population without including purely spatial clusters, during period 1, SaTScan identified the most likely statistically significant cluster $(\mathrm{RR}=185.3)$ including three SLAs within a period of $1 / 5 / 1993$ to $30 / 6 / 1993$. Another statistically significant secondary cluster $(R R=27.2)$ with six SLAs was also identified within the same time frame. Similarly, during period 2, SaTScan identified the most likely statistically significant cluster $(R R=55.8)$ with four
SLAs within a period of $1 / 1 / 1999$ to $31 / 12 / 2002$ and secondary cluster $(\mathrm{RR}=22.7)$ with three SLAs within the same time frame. Likewise, during period 3 SaTScan detected the most likely statistically significant cluster $(\mathrm{RR}=23.9)$ including 18 SLAs within a period of $1 / 1 / 2003$ to $31 / 12 / 2005$. Another secondary cluster ( $R R=6.8)$ consisting of 24 SLAs was also identified within a period of $1 / 1 / 2003$ to $31 / 12 / 2004$. During period 4 , a single large most likely statistically significant cluster $(\mathrm{RR}=22.8)$ consisting of 38 SLAs was identified within a period of $1 / 1 / 2009$ to $31 / 12 / 2009$. Another secondary cluster ( $R R=46.4)$ including 10 SLAs was also detected within a period of $1 / 1 / 2008$ to $31 / 12 / 2009$ (see Figure 3 ).

\begin{tabular}{|l|l|c|c|c|c|c|}
\hline Period & $\begin{array}{c}\text { C } \\
(\mathrm{n})\end{array}$ & $\begin{array}{c}\mathrm{R} \\
(\mathrm{km})\end{array}$ & $\mathrm{O}(\mathrm{n})$ & $\mathrm{E}(\mathrm{n})$ & $\mathrm{RR}$ & LLR \\
\hline $1993-$ & $1^{*}$ & 421 & 335 & 16 & 23.0 & 707.0 \\
2012 & $(16)$ & & & & & \\
& & & & & & \\
Sec & $1(2)$ & 4 & 505 & 88 & 7.00 & 504.2 \\
& $2(16)$ & 5 & 212 & 56 & 4.0 & 129.3 \\
& $3(4)$ & 35 & 101 & 34 & 3.02 & 43.1 \\
& $4(1)$ & 0 & 3 & 0 & 24.9 & 6.7 \\
\hline Period & $1^{*}(3)$ & 298 & 19 & 0.29 & 168. & 68.3 \\
1 & & & & & 8 & \\
& $2(6)$ & 3 & 10 & 0.83 & 17.4 & 17.3 \\
Period & $1^{*}(4)$ & 190 & 104 & 26.44 & 19.1 & 110.7 \\
2 & & & & & & \\
& $2(3)$ & 3 & 12 & 0.58 & 22.7 & 25.4 \\
Period & $1^{*}$ & 844 & 744 & 102.40 & 23.2 & 1136. \\
3 & $(18)$ & & & & & 5 \\
& $2(14)$ & 4 & 84 & 13.25 & 6.8 & 86.8 \\
Period & $3(3)$ & 33 & 4 & 0.40 & 10.1 & 5.6 \\
4 & $1^{*}$ & 596 & 1124 & 445.60 & 40.5 & 914.9 \\
& $(40)$ & & & & & \\
& $2(10)$ & 421 & 15 & 0.3 & 46.4 & 42.8 \\
\hline
\end{tabular}

$\mathrm{C}=$ Cluster $(\mathrm{n})=$ number of SLAs; $*$ * Primary cluster; $\mathrm{Sec}=$ Secondary cluster; $\mathrm{R}=$ Radius; $\mathrm{O}=$ Observed cases; $\mathrm{E}=$ Expected cases; $\mathrm{RR}=$ Relative risk; $\mathrm{LLR}=\mathrm{Log}$-likelihood ratio

Table 3: Spatial clusters of dengue identified using spatial scan test statistic

Table 4 shows the cluster, number of SLAs included in each cluster, radius $(\mathrm{km})$, observed cases, expected cases, relative risk and log-likelihood ratio for each time period. Very similar results were obtained when analysis was conducted using a spatial cluster size of $50 \%$ of population.

Both high-risk clusters detected in SaTScan were either included in or overlapped those identified using GeoDa.

\subsection{Discussion}

The results of this study revealed a significant spatio-temporal variation in the distribution of dengue in north Queensland, Australia. Previous studies showed that, in Queensland, the dengue epidemic often had a cycle of $5-6$ years and was expected to reach a 6-year peak (Hu et al., 2010, Hanna, 2009). These peaks were in 2003 and 2009, as also recorded in this study, showing an upward trend of dengue cases with the largest outbreak in 2009 (see Table 1). In our space-time clustering analysis, most of the dengue cases occurred during summer and autumn (i.e., the first quarter of the year), when the rainfall and temperature are highest of the year. Thus, high-risk transmission appeared to occur between these two seasons each year from 2003 to 2009 (see Table 4). Therefore, we can conclude that a clear seasonal pattern of dengue occurrence is observed in 
northern Queensland. These results are also consistent with those of the studies conducted in other tropical/sub-tropical environments. For example, some studies in Vietnam found that dengue was most prevalent in the wet season (Cuong, 2011, Pham et al., 2011, Toan, 2013).

\begin{tabular}{|c|c|c|c|c|c|c|c|}
\hline Period & $\begin{array}{l}\mathrm{C} \\
\text { (n) }\end{array}$ & $\begin{array}{l}\text { Time } \\
\text { frame }\end{array}$ & $\begin{array}{l}\text { R } \\
(\mathrm{km})\end{array}$ & $\begin{array}{l}\mathrm{O} \\
\text { (n) }\end{array}$ & $\begin{array}{l}\text { E } \\
\text { (n) }\end{array}$ & $\mathrm{RR}$ & LLR \\
\hline $\begin{array}{l}1993- \\
2012\end{array}$ & $\begin{array}{l}1^{*} \\
(26)\end{array}$ & $\begin{array}{l}1 / 3 / 03- \\
30 / 4 / 09\end{array}$ & 561 & 1584 & 350 & 11.7 & $\begin{array}{l}1652 . \\
8\end{array}$ \\
\hline \multirow[t]{2}{*}{$\mathrm{Sec}$} & $\begin{array}{l}1 \\
(19)\end{array}$ & $\begin{array}{l}1 / 4 / 02- \\
30 / 4 / 09\end{array}$ & 8 & 177 & 29 & 6.57 & 178.2 \\
\hline & $\begin{array}{l}2 \\
(2)\end{array}$ & $\begin{array}{l}1 / 1 / 09- \\
1 / 3 / 09\end{array}$ & 11 & 100 & 35 & 2.91 & 40.2 \\
\hline \multirow[t]{2}{*}{$\begin{array}{l}\text { Period } \\
1\end{array}$} & $\begin{array}{l}1^{*} \\
(3)\end{array}$ & $\begin{array}{l}1 / 5 / 93- \\
0 / 6 / 93\end{array}$ & 298 & 19 & 0.2 & $\begin{array}{l}185 . \\
3\end{array}$ & 70.0 \\
\hline & $\begin{array}{l}2 \\
(6)\end{array}$ & $\begin{array}{l}1 / 3 / 93- \\
1 / 7 / 93\end{array}$ & 3 & 10 & 0.5 & 27.2 & 21.5 \\
\hline \multirow[t]{2}{*}{$\begin{array}{l}\text { Period } \\
2\end{array}$} & $\begin{array}{l}1^{*} \\
(4)\end{array}$ & $\begin{array}{l}1 / 1 / 99- \\
31 / 12 / 02\end{array}$ & 190 & 104 & 10.5 & 55.8 & 203.1 \\
\hline & $\begin{array}{l}2 \\
(3)\end{array}$ & $\begin{array}{l}1 / 1 / 99- \\
31 / 2 / 02\end{array}$ & 3 & 12 & 0.5 & 22.7 & 25.4 \\
\hline \multirow[t]{2}{*}{$\begin{array}{l}\text { Period } \\
3\end{array}$} & $\begin{array}{l}1^{*} \\
(18)\end{array}$ & $\begin{array}{l}1 / 1 / 03- \\
2 / 31 / 05\end{array}$ & 844 & 738 & 97.2 & 23.9 & $\begin{array}{l}1154 . \\
3\end{array}$ \\
\hline & $\begin{array}{l}2 \\
(24)\end{array}$ & $\begin{array}{l}1 / 1 / 03- \\
2 / 31 / 04\end{array}$ & 159 & 76 & 11.8 & 6.8 & 79.1 \\
\hline \multirow[t]{2}{*}{$\begin{array}{l}\text { Period } \\
4\end{array}$} & $\begin{array}{l}1^{*} \\
(38)\end{array}$ & $\begin{array}{l}1 / 1 / 09- \\
12 / 31 / 09\end{array}$ & 596 & 916 & 159.6 & 22.8 & $\begin{array}{l}1250 . \\
1\end{array}$ \\
\hline & $\begin{array}{l}2 \\
(10)\end{array}$ & $\begin{array}{l}1 / 1 / 08- \\
12 / 31 / 09\end{array}$ & 421 & 15 & 0.3 & 46.4 & 42.8 \\
\hline
\end{tabular}

$\mathrm{C}=$ Cluster $(\mathrm{n})=$ number of SLAs; $*=$ Primary cluster; $\mathrm{Sec}=$ Secondary cluster; $\mathrm{R}=$ Radius; $\mathrm{O}=$ Observed cases; $\mathrm{E}=$ Expected cases; $\mathrm{RR}=$ Relative risk; $\mathrm{LLR}=\mathrm{Log}$-likelihood ratio

Table 4: Space-time clusters of dengue identified using spatial scan test statistic

The impact of rain on the dengue mosquito breeding and spread of the dengue has been documented in many studies. For example, Khormi et al (Khormi et al., 2011) pointed out that rainfall creates hotbeds for Ae. aegypti reproduction and increases the vegetation index. Generally, during the wet or monsoon season (i.e., from November to March), northern Queensland has a slightly higher temperature by a few degrees and higher humidity than during the hot dry season (i.e., from April to October). Moreover, the high humidity and high temperatures which characterise the climate in this part of Queensland region may have a positive impact on the survival and breeding conditions of mosquitoes.

Application of SaTScan method identified both purely spatial and space-time dengue high-risk clusters. It clearly demonstrated heterogeneity of dengue risk at the SLA level in Queensland and revealed the spatial and temporal pattern of dengue clusters across the northern region. Both purely spatial and space-time retrospective analyses showed the existence of most likely dengue clusters at the same SLAs (see Tables $3 \&$ 4). When the space-time analysis was modified to detect clusters with different population size, the same most likely cluster of $50 \%$ population size cluster remained significant for the years 2003 and 2009. Similarly, the results of Anselin's test revealed significant spatial and temporal variation in the distribution of dengue in northern Queensland.

Results from space-time clustering analysis identified the areas with most likely clusters over the larger region and over the study period (see Table 4). Using the maximum cluster size of $50 \%$ of the total population, we identified a single most likely cluster including 26 SLAs within a period of $1 / 3 / 2003$ to $30 / 4 / 2009$ where higher /medium population is concentrated on average. These 26 SLAs were as following: Aurukun, Weipa, Cook, Injinoo, Seisia, Bamaga, Torres, Hammond, St Pauls, Badu, Warraber, Poruma, Lama, Mareeba, Yorke, Mer, Douglas, Erub, Cairns - Pt A, Cairns - Barron, Cairns - Mt Whitfield, Cairns - Western suburbs, Cairns city - Northern suburbs, Cairns city - Trinity, Cairns - City and Cairns - Central suburbs. Furthermore, another 19 SLAs were detected as the secondary cluster during the period $1 / 4 / 2002$ to $30 / 4 / 2009$ and these were Cairns City, South Townsville, Northward - Castle hill, Railway estate, Randwick , Hermit park, Hyde parkMysterton, Pimlico, Rowes Bay-Belgian gardens, Currajong, Rosslea, Oonoonba-Idalia-Cluden, Garbutt, Gulliver, Heatley, Mundingburra, Vincent, Aitkenvale and Wulguru. Similar results were observed in Malaysia, where the spatial distribution of dengue was closely related to population density (Er, 2010). Therefore, spatial clustering of dengue is inevitable, since the human population usually live in spatial clusters rather than spatial randomness.

Clusters generated using a larger size scan window produce larger clusters which can help policy-makers to make decisions at larger geographic levels, for example, at the regional level. However, these large spatial clusters will cover a large area with a larger and more heterogeneous population. Conversely, clusters generated using smaller circular windows will produce smaller clusters but will contain a more homogeneous population which can help policy makers in planning more focused community interventions. For example, in this study, we used circular windows no more than $50 \%$ to identify dengue with most likely significant clusters and smaller circular windows of no more than $10 \%$ to identify possible secondary clusters for more efficient resource allocation for controlling and preventing dengue outbreaks.

Spatial autocorrelation is a valuable tool for examining the spatial pattern changes over time. In this study, we found that dengue had high spatial autocorrelation in four different time periods. The patterns were closely related to the geographical features of the study area, in that the SLAs closer together tend to have more similar incidence rates than farther SLAs.

Certain geographic features, such as, Julia creek and Torrens creek (northern Queensland), may not be considered, and cluster boundaries, due the number of overlapping circular windows, may not be well defined. Anselin's Local Moran test for spatial autocorrelation, on the other hand, is less sensitive to the unique geographic features of north Queensland, but examined clustering patterns according to user-defined neighbour relationships. Despite these different attributes, both cluster detection methods identified similar significant clustering. Consistent results using these two methods suggest that these findings are robust. Improving our understanding of the geographic distribution of dengue will ultimately provide crucial information on the role of environmental and/or sociocultural factors in the complex causal mechanisms underlying dengue etiology.

In this study, the reasons for clustering within the SLAs were not explored. However, certain assumptions could be made. The SLAs involved in the most likely cluster were located in the central, western and northern suburbs of Cairns. The Cairns region is one of the fastest growing regions in Australia, with 
more than $3 \%$ annual growth experienced over the last 10 years (Australian Bureau of Statistics, 2008). However, growth has slowed in recent years in response to global economic circumstances. Two thirds of tropical north Queensland's population growth (approximately 70,000 people) will be accommodated within the Cairns Region. Cairns city is the principal centre of the region and is centrally located along the coastal strip with sub-regions to the north and south consisting predominantly good quality agricultural land and areas of high ecological significance. As other tropical countries, tropical north Queensland's climate is favourable for the transmission of dengue (Australian Bureau of Meteorology, 2014). A warm temperature is crucial to the dengue mosquito's life and gonotrophic cycle, and to virus replication in tropical countries (Van Den Hurk et al., 2010). In addition, stagnant water and higher humidity could augment the epidemic during a rainy season (Pinto et al., 2011).

Statistically significant clustering at the SLA level, including in areas where dengue is epidemic, provides evidence that other co-factors may be involved. These findings may be either linked to unique geographic features of the area, related to shared socio-cultural practices or both. However, the region's peculiar ecological characteristics may also make a suitable geographic area for mosquito breeding. Cairns, for example, is famous for domestic and international tourism activities (Australian Bureau of Statistics, 2013) and economically developed region in Queensland. Furthermore, it is an important transport hub in the Far North Queensland region. Usually, all dengue outbreaks in Queensland were associated with viraemic travellers coming from a dengue endemic country, among which Bali in Indonesia, Papua New Guinea and India are ranked the highest (Knope et al., 2013). The high incidence of dengue in 2010 may be due to the development of Cairns International airport which may have attracted many overseas travellers (Queensland Treasury and Trade, 2013). Thus, clustering may result in increased mosquito populations and dengue transmission, and it may also be associated with temporary displacement of people. As suggested by Stoddard et al (Stoddarda et al., 2013), house to house human movements may underlie spatial clustering of dengue incidence causing marked heterogeneity in transmission rates. At a community level, transmission appears to be shaped by social connections because regular movements among the same places, such as the homes of family and friends, are often similar for the infected individual and their contacts. Furthermore, as suggested by previous study in Cairns (Duncombe, 2013), clustering may be due to a profusion of vector breeding sites in the high-risk areas. Dengue is not endemic in Queensland, however, Ae. aegypti is widespread throughout urban tropical north Queensland. The clustering indicates that these areas are perhaps persistent breeding areas and should be targeted mostly for mosquito control activities.

One of the strengths of this study is the use of both clustering methods to detect hot spots or high-risk areas of dengue in north Queensland. Using SaTScan enables us to analyse variations in any event of interest for many possible small and large geographic areas and without being restricted by administrative boundaries. In SaTScan, the shape of the scan window can be circular or elliptical. Circular windows provide good level of accuracy if the population at risk exists in circular shaped areas. However, as the population at risk do not exist in circular shaped areas, elliptical-shaped scan windows will provide slightly increased power for identifying non-circular shaped clusters (e.g., long narrow clusters). Tango and Takahashi recently (Tango, 2005) proposed using flexible shaped spatial scan statistics to accommodate irregular shaped clusters. Another advantage is that where point data are not available, the centroids of the smallest geographical spatial unit that are available can be used in SaTScan (Kulldorff, 1997). Lastly, SaTScan is also capable of performing space-time scan statistics to identify clusters existing in both space and time.

To the best of our knowledge, this is the first study to apply both Kulldorff's SaTScan and Anselin's local Moran test, to investigate the geographical clustering of dengue in northern Queensland, Australia. Both methods detected similar and statistically significant high-risk clustering, suggesting that our results are robust (see Figures $2 \& 3$ ).

This study also has limitations. First, our surveillance may have missed some dengue cases in Queensland. However, the underreporting is likely to be random both in location and in demographic characteristics due to the large efforts and attention for the dengue surveillance system to cover all laboratory-diagnosed cases with medical services. Second, the use of place of residence may not be proper, as it might not capture the exposure or infection; but because of the missing information of place of work from the surveillance data, we could not include it as a routine reported variable to capture work exposure. A third limitation is that we did not explore the causes of these clusters in our analyses. Despite these limitations, this study detected spatial and space -time clusters of dengue in tropical north Queensland, and these findings may have significant implications for controlling and preventing dengue outbreaks.

\section{ACKNOWLEDGEMENTS}

$\mathrm{S} \mathrm{N}$ would like to acknowledge ISPRS travel grant and QUT.

\section{REFERENCES}

Anselin, L. 1995. Local indicators of spatial association-LISA. Geograph Anal, 27, 93-115.

Anselin, L. 2004. Review of cluster analysis software. Report in fulfillment of consultant agreement with the North American Association of Central Cancer Registries Inc.

Australian Bureau of Meteorology. 2014. Data obtained from National climate centre, Queensland, Commonwealth of Australia [Online]. Available: www.bom.gov.au/climate/ [Accessed 10 March, 2014].

Australian Bureau of Statistics. 2008. Population projections Australia 2004 to 2101 (cat. no. 3222.0) [Online]. Available: www.abs.gov.au [Accessed August 29, 2014].

Australian Bureau of Statistics. 2013. Census of Population and Housing - Basic community profiles, Commonwealth of Australia, Canberra, ABS [Online]. Available: www.oesr.qld.gov.au [Accessed 29 May 2014].

Bhatt, S., et al. 2013. The global distribution and burden of dengue. Nature, 496, 504-507.

Caminade, C., et al. 2012. Suitability of European climate for the Asian tiger mosquito Aedes albopictus: recent trends and future scenarios. $J R$ Soc Interface, 1-11.

Cdc. 2014. Dengue and Climate [Online]. Centers for Disease Control and Prevention, Communicable Diseases Center. Available:

http://www.cdc.gov/dengue/entomologyEcology/climate.html [Accessed 30 April 2014]. 
Chen, J., et al. 2008. Geovisual analytics to enhance spatial scan statistic interpretations: an analysis of US cervical cancer mortality. Int J Health Geogr 7, 18.

Coleman, M., et al. 2009. Using the SaTScan method to detect local malaria clusters for guiding malaria control programmes. Malar J, 8, 68-74.

Cuong, H. Q., Hien, N.T., Duong, T.N., Phong, T.V., Cam, N.N., Et Al. 2011. Quantifying the Emergence of Dengue in Hanoi, Vietnam: 1998-2009. PLoS Negl Trop Dis, 5, e1322.

Duncombe, J., Clements, A., Davis, J., Hu, W., Weinstein, P., Ritchie, S. 2013. Spatiotemporal patterns of Aedes aegypti populations in Cairns, Australia: assessing drivers of dengue transmission. Tro Med Int Health, 18, 839-849.

Er, A., Rosli Mh, Asmahani a, Mohamad Naim Mr, Harsuzilawati M 2010. Spatial mapping of dengue incidence: A case study in Hulu Langat District, Selangor, Malaysia. Int $J$ Hum Soc Sci, 5, 410-414.

Estallo, E. L., et al. 2011. Prevention of dengue outbreaks through Aedes aegypti oviposition activity forecasting method. Vector Borne Zoonotic Dis, 11, 543-9.

Hanna, J. N., Ritchie, S.A. 2009. Outbreaks of dengue in north Queensland, 1990-2008. Communicable Diseases Intelligence, 33, 32-33.

Higa, Y. 2011. Dengue Vectors and their Spatial Distribution. Trop Med Health, 39, 17-27.

Honorio, N. A., et al. 2009. The spatial distribution of Aedes aegypti and Aedes albopictus in a transition zone, Rio de Janeiro, Brazil. Cad Saude Publica, 25, 1203-14.

$\mathrm{Hu}$, W., et al. 2010. Dengue fever and El Nino/Southern Oscillation in Queensland, Australia: a time series predictive model. Occup Environ Med, 67, 307-11.

Impoinvil, D. E., et al. 2011. The Spatial Heterogeneity between Japanese Encephalitis Incidence Distribution and Environmental Variables in Nepal. PLoS ONE, 6, e22192.

Jansen, C. C., et al. 2010. The dengue vector Aedes aegypti: what comes next. Microbes Infect, 12, 272-9.

Khormi, H. M., et al. 2011. Describing and analysing the association between meteorological variables and adult Aedes aegypti mosquitoes. J Food Agr Environ, 9, 954-959.

Knope, K., et al. 2013. Increasing notifications of dengue in Australia related to overseas travel, 1991 to 2012. Commun Dis Intell, 37, E5-E7.

Kulldorff, M. 1997. A spatial scan statistic. Communications in Statistics: Theory and Methods, 26, 1487-1496.

Kulldorff, M. 2009. SatScan Version 8.02: Software for the Spatial and Space-Time Scan Statistics. Silver Spring, MD: Information Management Services.

Moore, D. A., et al. 1999. Spatial analytical methods and geographic information systems : Use in health research and epidemiology. Epidemiol Rev, 21, 143-161.

Naish, S., et al. 2011. Spatial and temporal clusters of Barmah Forest virus disease in Queensland, Australia. Trop Med Int Health, 16, 884-893.

National Notificable Disease Surveillance System, N. 2014. Number of notifications of dengue virus infection received from State and Territory health authorities in the period of 1991 to 2012 and year-to-date notifications for 2013 [Online]. Department of Health and Ageing. Available: http://www9.health.gov.au/cda/source/Rpt_4.cfm [Accessed 16 April 2014].

Pham, H. V., et al. 2011. Ecological factors associated with dengue fever in a Central Highlands province, Vietnam. BMC Infect Dis, 11, 172.

Pinto, E., et al. 2011. The influence of climate variables on dengue in Singapore. Int J Environ Health Res, 21, 415-26.
Queensland Health. 2013. Mosquito-borne diseases [Online]. Brisbane, Queensland: Queensland Health, National Notifiable Diseases Surveillance System, Communicable Diseaes Branch. Available: www.health.qld.gov.au [Accessed 20 November 2013].

Queensland Health. 2014. Dengue fever [Online]. Brisbane: Queensland Health. Available: http://www.health.qld.gov.au/dengue/outbreaks/current.asp [Accessed 20 March 2014].

Queensland Treasury and Trade. 2013. Population growth highlights and trends, Queensland [Online]. Government Statistician. Available: http://www.oesr.qld.gov.au/products/publications/pop-growthhighlights-trends-qld/pop-growth-highlights-trends-qld-

2013.pdf [Accessed 10 August 2013].

Russell, R. C. 1998. Vectors vs. humans in Australia--who is on top down under? An update on vector-borne disease and research on vectors in Australia. J Vector Ecol, 23, 1-46.

Russell, R. C. 2009a. Mosquito-borne disease and climate change in Australia: time for a reality check. Aust J Entomol, 48, 1-7.

Russell, R. C., Currie, B. J.,Lindsay, M.D., Mackenzie, J.S., Ritchie, S.A., Whelan, P.I. 2009b. Dengue and climate change in Australia: predictions for the future should incorporate knowledge from the past. Med J Aust, 190, 265-268.

Stoddarda, S. T., et al. 2013. House-to-house human movement drives dengue virus transmission. Proc Natl Acad Sci U S A, 110, 994-999.

Takahashi, L. T., et al. 2005. Mathematical models for the Aedes aegypti dispersal dynamics: travelling waves by wing and wind. Bull Math Biol, 67, 509-28.

Tango, T., Takahashi, K. 2005. A flexibly shaped spatial scan statistisc for detecting clusters. Int J Health Geogr, 4, 1-15.

Toan, D. T. T., Hu, W., Thai,P.Q., Hoat,L.N., Wright, P., Martens, P. 2013. Hot spot detection and spatio-temporal dispersion of dengue fever in Hanoi, Vietnam. Global Health Action, 6, 10-18.

Van Den Hurk, A. F., et al. 2010. Emerging tropical diseases in Australia. Part 4. Mosquitoborne diseases. Ann Tro Med Parasitol, 104, 623-640.

Vanwambeke, S. O., et al. 2011. Spatially disaggregated disease transmission risk: land cover, land use and risk of dengue transmission on the islaand of Oahu. Tropical Medicine and International Health, 16, 174-185.

Vezzani, D., et al. 2008. Aedes aegypti, Aedes albopictus, and dengue in Argentina: current knowledge and future directions. Memórias Do Instituto Oswaldo Cruz, 103, 66-74.

Wen, T. H., et al. 2010. Spatial-temporal patterns of dengue in areas at risk of dengue haemorrhagic fever in Kaohsiung, Taiwan, 2002. International Journal of Infectious Diseases, 14, e334-e343.

Williams, C. R., et al. 2010 The Extinction of Dengue through Natural Vulnerability of Its Vector. PLoS Negl Trop Dis 4, e922.

World Health Organisation. 2014. Better environmental management for control of dengue [Online]. Available: http://www.who.int/heli/risks/vectors/denguecontrol/en/ [Accessed 20 June 2014]. 